\title{
WELLORDERING PROOFS FOR METAPREDICATIVE MAHLO
}

\author{
THOMAS STRAHM
}

\begin{abstract}
In this article we provide wellordering proofs for metapredicative systems of explicit mathematics and admissible set theory featuring suitable axioms about the Mahloness of the underlying universe of discourse. In particular, it is shown that in the corresponding theories EMA of explicit mathematics and $\mathrm{KPm}^{0}$ of admissible set theory, transfinite induction along initial segments of the ordinal $\varphi \omega 00$, for $\varphi$ being a ternary Veblen function, is derivable. This reveals that the upper bounds given for these two systems in the paper Jäger and Strahm [11] are indeed sharp.
\end{abstract}

§1. Introduction. This paper is a companion to the article by Jäger and Strahm [11], where systems of explicit mathematics and admissible set theory for metapredicative Mahlo are introduced. Whereas the main concern of [11] was to establish proof-theoretic upper bounds for these systems, in this article we provide the corresponding wellordering proofs, thus showing that the upper bounds derived in [11] are sharp.

The central systems of this article are the theories EMA and $\mathrm{KPm}^{0}$ for metapredicative Mahlo in explicit mathematics and admissible set theory, respectively. EMA is based on Feferman's explicit mathematics with elementary comprehension and join (c.f., Feferman [2, 3]). Crucial for its formulation are so-called universes: these are types of representations or names which are closed under elementary comprehension and join. The principal axiom of EMA claims that for each operation from names of types to names of types there exists a uniformly given universe that is closed under this operation. We note that EMA does not include inductive generation and that induction on the natural number is restricted to types. For more information concerning EMA plus inductive generation see Jäger and Studer [12].

The theory $\mathrm{KPm}^{0}$, on the other hand, is Rathjen's theory KPM (c.f., Rathjen [16, 17]) with induction on the natural numbers restricted to sets and $\epsilon$ induction omitted completely. The Mahlo axiom schema in $\mathrm{KPm}^{0}$ features $\Pi_{2}$ reflection on admissible sets. It happens that the absence of $\in$ induction causes a dramatic collapse in proof-theoretic strength: whereas KPM is a highly impredicative theory exceeding $\left(\Delta_{2}^{1}-\mathrm{CA}\right)+(\mathrm{BI})$ in proof strength by far, the strength of $\mathrm{KPm}^{0}$ is between the Feferman-Schütte ordinal $\Gamma_{0}$ and the Bachmann-Howard ordinal.

The theories EMA and $\mathrm{KPm}^{0}$ and their proof-theoretic analyses typically belong to the new area of so-called metapredicative proof theory. Metapredicativity is concerned with the study and analysis of formal systems whose proof-theoretic

Received January 20, 2000; accepted February 20, 2001. 
ordinal is beyond $\Gamma_{0}$, but which can nevertheless be given a proof-theoretic analysis that uses methods from predicative proof theory only. Quite recently, numerous interesting metapredicative systems have been identified, c.f., e.g., $[8,10,13,18,19$, $23,22,20,21,27,26]$.

The term metapredicative indeed also applies to the wellordering proofs for EMA and $\mathrm{KPm}^{0}$ given in this paper. First of all, the notation system used is based on a ternary Veblen or $\varphi$ function $\varphi \alpha \beta \gamma$, which is a straightforward generalization of the well-known binary $\varphi$ function; in particular, no collapsing is used in this notation system. Secondly and most importantly, the general methodology of the wellordering proofs given below is very much in the spirit of the wellordering proofs for predicative systems due to Feferman and Schütte, c.f., e.g., [4, 5, 25]. For example, instead of working in initial segments of the ramified analytic hierarchy or the ordinary jump hierarchy one considers hierarchies of universes, hyperuniverses, admissibles, hyperinaccessibles, and so on.

The plan of this paper is as follows. In the next section we review the system EMA introduced in [11] and we identify its crucial subsystems $S_{n}(n \in \mathbb{N})$. The principal universe generation axiom of $S_{n}$ features the existence of so-called $n$-hyperuniverses, which can be seen as an analogue of $n$-(hyper)inaccessibles. Section 3 constitutes the heart of this article: after some ordinal-theoretic preliminaries we show that $\mathrm{S}_{n}$ derives transfinite induction along all initial segments of the ordinal $\varphi(n+1) 00$, thus establishing $\varphi \omega 00$ as a lower bound of EMA. In Section 4 we indicate how the wellordering proofs given for EMA can be adapted to the framework of admissible set theory, namely the theory $\mathrm{KPm}^{0}$. We will end our paper in Section 5 with some remarks concerning the strength of our theories in the presence of the full schema of complete induction on the natural numbers: it turns out that the methods of this paper readily yield that the ordinal $\varphi \varepsilon_{0} 00$ is a lower bound of EMA and $\mathrm{KPm}^{0}$ augmented by formula induction.

§. The theory EMA and its subsystems. In this section we recapitulate the theory EMA for metapredicative Mahlo in explicit mathematics with universes, which has been introduced in Jäger and Strahm [11]. Universes are types of (names of) types which are closed under elementary comprehension and join (disjoint union). The principal type existence axiom of EMA claims that each total operation on types (names) can be (uniformly) reflected in a universe. Furthermore, we identify crucial subsystems $S_{n}(n \in \mathbb{N})$ of EMA which are suited for carrying through the wellordering proofs in the next section.

Large parts of the first paragraph of this section are very much like in related papers; nevertheless, we decided to include them in order to make our article self-contained and also accessible for a reader who is not a specialist in explicit mathematics.

2.1. Defining EMA. EMA is formulated in the second order language $\mathbb{L}$ for individuals and types. It comprises individual variables $a, b, c, f, g, h, u, v, w, x, y, z, \ldots$ as well as type variables $U, V, W, X, Y, Z, \ldots$ (both possibly with subscripts). $\mathbb{L}$ also includes the individual constants $k, s$ (combinators), $p, p_{0}, p_{1}$ (pairing and projections), 0 (zero), $\mathrm{s}_{\mathrm{N}}$ (successor), $\mathrm{p}_{\mathrm{N}}$ (predecessor), $\mathrm{d}_{\mathrm{N}}$ (definition by numerical cases) and additional individual constants, called generators, which will be used for the uniform naming of types, namely nat (natural numbers), id (identity), co 
(complement), int (intersection), dom (domain), inv (inverse image), $\mathrm{j}$ (join) and $\mathrm{m}$ (universe generator). There is one binary function symbol · for (partial) application of individuals to individuals. Further, $\mathbb{L}$ has unary relation symbols $\downarrow$ (defined) and $\mathrm{N}$ (natural numbers) as well as three binary relation symbols $\in$ (membership), = (equality) and $\Re$ (naming, representation).

For a uniform definition of the notion of proof-theoretic ordinal (c.f., Jäger and Strahm [11], Definition 1) it is convenient that $\mathbb{L}$ also includes an anonymous unary relation symbol $Q$ and a corresponding generator $q$. The relation $Q$ plays the role of an anonymous predicate on the natural numbers with no specific mathematical meaning.

The individual terms $\left(r, s, t, r_{1}, s_{1}, t_{1}, \ldots\right)$ of $\mathbb{L}$ are built up from individual variables and individual constants by means of our function symbol f for application. In the following we often abbreviate $(s \cdot t)$ simply as $(s t)$, st or sometimes also $s(t)$; the context will always ensure that no confusion arises. We further adopt the convention of association to the left so that $s_{1} s_{2} \ldots s_{n}$ stands for $\left(\ldots\left(s_{1} \cdot s_{2}\right) \ldots s_{n}\right)$. We also set $t^{\prime}:=\mathrm{s}_{\mathrm{N}} t$. Finally, we define general $n$ tupling by induction on $n \geq 2$ as follows:

$$
\left(s_{1}, s_{2}\right):=\mathrm{p} s_{1} s_{2}, \quad\left(s_{1}, \ldots, s_{n+1}\right):=\left(\left(s_{1}, \ldots, s_{n}\right), s_{n+1}\right) .
$$

The positive literals of $\mathbb{L}$ are of the form $\mathrm{N}(s), s \downarrow, s=t, U=V, s \in U$ and $\Re(s, U)$. Since we work with a logic of partial terms, it is not guaranteed that all terms have values, and $s \downarrow$ is read as $s$ is defined. Moreover, $N(s)$ says that $s$ is a natural number, and the formula $\Re(s, U)$ is used to express that the individual $s$ represents the type $U$ or is a name of $U$.

The formulas $\left(A, B, C, A_{1}, B_{1}, C_{1}, \ldots\right)$ of $\mathbb{L}$ are generated from the positive literals by closing against the usual propositional connectives, as well as existential and universal quantification for individuals and types. The following table contains a useful list of abbreviations:

$$
\begin{aligned}
s \simeq t & :=s \downarrow \vee t \downarrow \rightarrow s=t, \\
s \in \mathrm{N} & :=\mathrm{N}(s), \\
(\exists x \in \mathrm{N}) A(x) & :=(\exists x)(x \in \mathrm{N} \wedge A(x)), \\
(\forall x \in \mathrm{N}) A(x) & :=(\forall x)(x \in \mathrm{N} \rightarrow A(x)), \\
U \subset V & :=(\forall x)(x \in U \rightarrow x \in V), \\
s \dot{\in} t & :=(\exists X)(\Re(t, X) \wedge s \in X), \\
(\exists x \dot{\in} s) A(x) & :=(\exists x)(x \dot{\in} \wedge A(x)), \\
(\forall x \dot{\in} s) A(x) & :=(\forall x)(x \dot{\in} \rightarrow A(x)), \\
s \doteq t & :=(\exists X)[\Re(s, X) \wedge \Re(t, X)], \\
s \dot{\subset} t & :=(\exists X, Y)[\Re(s, X) \wedge \Re(t, Y) \wedge X \subset Y], \\
\Re(s) & :=(\exists X) \Re(s, X) .
\end{aligned}
$$

The vector notation $\vec{U}$ and $\vec{s}$ is sometimes used to denote finite sequences of type variables $U_{1}, \ldots, U_{m}$ and individual terms $s_{1}, \ldots, s_{n}$, respectively, whose length is given by the context.

The logic of EMA is Beeson's classical logic of partial terms (c.f., Beeson [1] or Troelstra and Van Dalen [28]) for the individuals and classical logic with equality for the types. Observe that Beeson's formalization includes the usual strictness axioms. 
Now let us first introduce the auxiliary theory EETJ, which provides a framework for explicit elementary types with join. The nonlogical axioms of EETJ can be divided into the following groups I-IV:

I. Applicative axioms. These axioms formalize that the individuals form a partial combinatory algebra, that we have pairing and projection and the usual closure conditions on the natural numbers plus definition by numerical cases.

(1) $\mathrm{k} u v=u$,

(2) $\operatorname{suv} \downarrow \wedge \mathrm{suvw} \simeq u w(v w)$,

(3) $\mathrm{p}_{0}(u, v)=u \wedge \mathrm{p}_{1}(u, v)=v$,

(4) $0 \in \mathrm{N} \wedge(\forall x \in \mathrm{N})\left(x^{\prime} \in \mathrm{N}\right)$,

(5) $(\forall x \in N)\left(x^{\prime} \neq 0 \wedge \mathrm{p}_{\mathrm{N}}\left(x^{\prime}\right)=x\right)$,

(6) $(\forall x \in \mathrm{N})\left(x \neq 0 \rightarrow \mathrm{p}_{\mathrm{N}} x \in \mathrm{N} \wedge\left(\mathrm{p}_{\mathrm{N}} x\right)^{\prime}=x\right)$,

(7) $u \in \mathrm{N} \wedge v \in \mathrm{N} \wedge u=v \rightarrow \mathrm{d}_{\mathrm{N}} x y u v=x$,

(8) $u \in \mathrm{N} \wedge v \in \mathrm{N} \wedge u \neq v \rightarrow \mathrm{d}_{\mathrm{N}} x y u v=y$.

II. Explicit representation and extensionality. The following axioms state that each type has a name, that there are no homonyms and that equality of types is extensional.

(1) $(\exists x) \Re(x, U)$,

(2) $\Re(u, U) \wedge \Re(u, V) \rightarrow U=V$,

(3) $(\forall x)(x \in U \leftrightarrow x \in V) \rightarrow U=V$.

III. Basic type existence axioms. In the following we provide a finite axiomatization of uniform elementary comprehension plus join.

Natural numbers

$\Re($ nat $) \wedge(\forall x)(x \dot{\in}$ nat $\leftrightarrow \mathrm{N}(x))$.

Representation of $\mathrm{Q}$

$\Re(\mathbf{q}) \wedge(\forall x)(x \dot{\in} \mathbf{q} \leftrightarrow \mathrm{Q}(x)) \wedge \mathrm{q} \dot{\subset}$ nat.

Identity

$\Re($ id $) \wedge(\forall x)(x \dot{\in}$ id $\leftrightarrow(\exists y)(x=(y, y)))$.

Complements

$\Re(u) \rightarrow \Re(\operatorname{co}(u)) \wedge(\forall x)(x \dot{\epsilon} \operatorname{co}(u) \leftrightarrow x \dot{\notin} u)$.

Intersections

$\Re(u) \wedge \Re(v) \rightarrow \Re(\operatorname{int}(u, v)) \wedge(\forall x)(x \dot{\epsilon} \operatorname{int}(u, v) \leftrightarrow x \dot{\in} u \wedge x \dot{\epsilon} v)$.

Domains

$\Re(u) \rightarrow \Re(\operatorname{dom}(u)) \wedge(\forall x)(x \dot{\epsilon} \operatorname{dom}(u) \leftrightarrow(\exists y)((x, y) \dot{\epsilon} u))$.

Inverse images

$\Re(u) \rightarrow \Re(\operatorname{inv}(u, f)) \wedge(\forall x)(x \dot{\in} \operatorname{inv}(u, f) \leftrightarrow f x \dot{\in} u)$.

Joins

$\Re(u) \wedge(\forall x \dot{\in} u) \Re(f x) \rightarrow \Re(\mathrm{j}(u, f)) \wedge \Sigma(u, f, \mathrm{j}(u, f))$. 
In this last axiom the formula $\Sigma(u, f, v)$ expresses that $v$ names the disjoint union of $f$ over $u$, i.e.,

$$
\Sigma(u, f, v):=(\forall x)(x \dot{\epsilon} v \leftrightarrow(\exists y, z)(x=(y, z) \wedge y \dot{\epsilon} u \wedge z \dot{\epsilon} f y)) .
$$

IV. Uniqueness of generators. These axioms essentially guarantee that different generators create different names. To achieve this, we have for syntactically different generators $r_{0}$ and $r_{1}$ and arbitrary generators $s$ and $t$ :

(1) $r_{0} \neq r_{1}$,

(2) $(\forall x)(s x \neq t)$,

(3) $(\forall x, y)(s x=t y \rightarrow s=t \wedge x=y)$.

As usual, the axioms of a partial combinatory algebra allow one to define $\lambda$ abstraction and to prove a recursion or fixed point theorem. For proofs of these standard results the reader is referred to $[1,2]$.

Lemma 2.1 (Abstraction and recursion). 1. For each $\mathbb{L}$ term $t$ and all variables $x$ there exists an $\mathbb{L}$ term $(\lambda x . t)$ whose variables are those of $t$, excluding $x$, so that $\mathrm{EETJ}$ proves

$$
(\lambda x . t) \downarrow \wedge(\lambda x . t) x \simeq t .
$$

2. There exists a closed $\mathbb{L}$ term rec so that EETJ proves

$$
\operatorname{rec} f \downarrow \wedge \operatorname{rec} f x \simeq f(\operatorname{rec} f) x .
$$

In the original formulation of explicit mathematics, elementary comprehension is not dealt with by a finite axiomatization but directly as an infinite axiom scheme. An $\mathbb{L}$ formula is called elementary if it contains neither the relation symbol $\Re$ nor bound type variables. The following result of Feferman and Jäger [6] shows that this scheme of uniform elementary comprehension is provable from our finite axiomatization. Join and uniqueness of generators are not needed for this argument.

LEMMA 2.2 (Elementary comprehension). For every elementary formula $A\left(u, \vec{v}, W_{1}, \ldots, W_{n}\right)$ with at most the indicated free variables there exists a closed term $t$ of $\mathbb{L}$ so that $\mathrm{EETJ}$ proves:

1. $\bigwedge_{i=1}^{n} \Re\left(w_{i}, W_{i}\right) \rightarrow \Re\left(t\left(\vec{v}, w_{1}, \ldots, w_{n}\right)\right)$,

2. $\bigwedge_{i=1}^{n} \Re\left(w_{i}, W_{i}\right) \rightarrow(\forall x)\left(x \dot{\epsilon} t\left(\vec{v}, w_{1}, \ldots, w_{n}\right) \leftrightarrow A\left(x, \vec{v}, W_{1}, \ldots, W_{n}\right)\right)$.

Let us now introduce the concept of a universe into explicit mathematics. To put it very simply, a universe is supposed to be a type which consists of names only and reflects the theory EETJ.

For the detailed definition of a universe we introduce some auxiliary notation and let $\mathscr{C}(W, u)$ be the closure condition which is the disjunction of the following $\mathbb{L}$ formulas:

(1) $u=$ nat $\vee u=\mathrm{q} \vee u=\mathrm{id}$,

(2) $(\exists x)(u=\operatorname{co}(x) \wedge x \in W)$,

(3) $(\exists x, y)(u=\operatorname{int}(x, y) \wedge x \in W \wedge y \in W)$,

(4) $(\exists x)(u=\operatorname{dom}(x) \wedge x \in W)$,

(5) $(\exists x, f)(u=\operatorname{inv}(x, f) \wedge x \in W)$,

(6) $(\exists x, f)[u=\mathrm{j}(x, f) \wedge x \in W \wedge(\forall y \dot{\in} x)(f y \in W)]$.

Thus, the formula $(\forall x)(\mathscr{C}(W, x) \rightarrow x \in W)$ states that $W$ is a type which is closed under the type constructions of EETJ, i.e., elementary comprehension and 
join. If, in addition, all elements of $W$ are names, we call $W$ a universe, in symbols, $\mathrm{U}(W)$. Moreover, we write $\mathscr{U}(u)$ to express that the individual $u$ is the name of a universe.

$$
\begin{aligned}
\mathrm{U}(W) & :=(\forall x)(\mathscr{C}(W, x) \rightarrow x \in W) \wedge(\forall x \in W) \Re(x), \\
\mathscr{U}(u) & :=(\exists X)(\Re(u, X) \wedge \mathrm{U}(X)) .
\end{aligned}
$$

Based on (names of) universes we can now introduce the Mahlo axiom for explicit mathematics. Given a name $x$ and an operation $f$ from names to names one simply claims that there exists (a name of) a universe $\mathrm{m}(x, f)$ which contains $x$ and reflects $f$.

The following shorthand notations are useful for obtaining a compact form of our Mahlo axiom:

$$
\begin{aligned}
(f: \Re \rightarrow \Re) & :=(\forall x)(\Re(x) \rightarrow \Re(f x)), \\
(f: s \rightarrow s) & :=(\forall x \dot{\in} s)(f x \dot{\in} s) .
\end{aligned}
$$

Mahloness in explicit mathematics is now expressed by the axioms

$$
\begin{aligned}
& \Re(x) \wedge(f: \Re \rightarrow \Re) \rightarrow \mathscr{U}(\mathrm{m}(x, f)) \wedge x \dot{\epsilon} \mathrm{m}(x, f), \\
& \Re(x) \wedge(f: \Re \rightarrow \Re) \rightarrow(f: \mathrm{m}(x, f) \rightarrow \mathrm{m}(x, f)) .
\end{aligned}
$$

It is interesting to examine what kind of ordering principles for universes can be consistently added to the previous axioms. This question is discussed at full length in Jäger, Kahle and Studer [9], and it is shown there that one must not be too liberal. As a consequence of these considerations we do not claim linearity and connectivity for arbitrary universes, but only for so-called normal universes, i.e., universes which are named by means of the type generator $m$,

$$
\mathscr{U}_{\mathrm{m}}(u):=(\exists x, f)[u=\mathrm{m}(x, f) \wedge \mathscr{U}(u)] .
$$

Linearity and connectivity of normal universes are then given by the following two axioms:

$$
\begin{aligned}
& \left(\mathscr{U}_{\mathrm{m}} \text {-Lin }\right)(\forall x, y)\left[\mathscr{U}_{\mathrm{m}}(x) \wedge \mathscr{U}_{\mathrm{m}}(y) \rightarrow x \dot{\epsilon} y \vee x \doteq y \vee y \dot{\epsilon} x\right], \\
& \left(\mathscr{U}_{\mathrm{m}} \text {-Con }\right)(\forall x, y)\left[\mathscr{U}_{\mathrm{m}}(x) \wedge \mathscr{U}_{\mathrm{m}}(y) \rightarrow x \dot{\subset} y \vee y \dot{\subset} x\right] .
\end{aligned}
$$

It is shown in [9] that connectivity of normal universes also implies transitivity of normal universes in its most general form. For the reader's convenience we briefly sketch the relevant argument.

LEMMA 2.3 (Strong transitivity). We have that EETJ $+\left(\mathscr{U}_{\mathrm{m}}\right.$-Con) proves

$$
\mathscr{U}_{\mathrm{m}}(u) \wedge \mathscr{U}_{\mathrm{m}}(v) \wedge w \doteq u \wedge w \dot{\epsilon} v \rightarrow u \dot{\subset} v .
$$

Proof. Assume the premise of the implication to be proved. Then $w$ is also a name of the universe named by $u$. Since universes never contain their names (c.f., e.g., Marzetta [14]) we have $w \dot{\notin} u$, thus $v \not \subset u$. But now connectivity of normal universes $\left(\mathscr{U}_{\mathrm{m}}\right.$-Con) yields $u \dot{\subset} v$ as desired.

The last principle present in EMA is complete induction on the natural numbers for types. Accordingly, type induction $\left(T-I_{N}\right)$ is the axiom

$$
(\forall X)\left(0 \in X \wedge(\forall x \in \mathrm{N})\left(x \in X \rightarrow x^{\prime} \in X\right) \rightarrow(\forall x \in \mathrm{N})(x \in X)\right) .
$$


To sum up, the theory EMA of explicit mathematics, whose universe is Mahlo, comprises the theory EETJ plus the Mahlo axioms (M.1) and (M.2), the ordering principles $\left(\mathscr{U}_{m}-\mathrm{Lin}\right)$ and $\left(\mathscr{U}_{m}-\right.$ Con) as well as type induction $\left(\mathrm{T}-\mathrm{I}_{\mathrm{N}}\right)$.

2.2. The subsystems $S_{n}$ of EMA. The crucial type existence axiom of $S_{n}$ claims the existence of $n$-hyperuniverses, which can be seen as an analogue of $n$-(hyper)inaccessible sets. We will see that the existence of $n$-hyperuniverses for each natural number $n$ is an immediate consequence of the Mahlo axioms (M.1) and (M.2). Moreover, the wellordering proofs in the next section will reveal that the prooftheoretic strength of EMA is already exhausted by its subsystems $S_{n}$ for each $n \in \mathbb{N}$.

For the formulation of $S_{n}$ we augment our language $\mathbb{L}$ by a generator constant $u_{n}$ for each natural number $n$. Below we define the notion of a type $W$ being an $n$-hyperuniverse, $n-U(W)$; accordingly, $n-\mathscr{U}(u)$ expresses that $u$ is the name of an $n$-hyperuniverse,

$$
\begin{aligned}
0-\mathrm{U}(W) & :=\mathrm{U}(W), \\
(n+1)-\mathrm{U}(W) & :=\mathrm{U}(W) \wedge(\forall x \in W)\left(\mathrm{u}_{n}(x) \in W\right), \\
n-\mathscr{U}(u) & :=(\exists X)(\Re(u, X) \wedge n-\mathrm{U}(X)) .
\end{aligned}
$$

The defining axiom for the constant $\mathrm{u}_{n}$ claims for each name $x$ that $\mathrm{u}_{n}(x)$ is the name of an $n$-hyperuniverse containing $x$,

$$
\Re(x) \rightarrow n-\mathscr{U}\left(\mathrm{u}_{n}(x)\right) \wedge x \dot{\epsilon} \mathrm{u}_{n}(x) .
$$

The theory $S_{n}$ now extends elementary explicit type theory with join EETJ by (i) the defining axioms for the constants $\mathrm{u}_{m}(m \leq n)$, (ii) linearity and connectivity axioms for universes which are normal with respect to the generators $\mathrm{u}_{m}(m \leq n)$, and (iii) type induction $\left(T-I_{N}\right)$ on the natural numbers.

We observe that due to the presence of the linearity and connectivity axioms for normal universes in $S_{n}$, we also have strong transitivity for such universes according to (the proof of) Lemma 2.3 above.

LEMMA 2.4 ( $n$-hyperuniverses in EMA). We have that $\mathrm{S}_{n}$ is contained in EMA for each natural number $n$.

Proof. The type generators $u_{n}$ can be defined in EMA by means of $m$,

$$
\mathrm{u}_{0}=\lambda x \cdot \mathrm{m}(x, \lambda y \cdot y), \quad \mathrm{u}_{n+1}=\lambda x \cdot \mathrm{m}\left(x, \mathrm{u}_{n}\right) .
$$

One readily shows by induction on $n$ and by making use of the Mahlo axioms (M.1) and (M.2) that the so-defined $u_{n}$ 's satisfy their defining axioms in $S_{n}$. In the case $n=0$ we have that $\mathrm{u}_{0}(x)$ is a universe containing $x$ for each name $x$, since trivially $(\lambda y, y)$ is a total operation from $\Re$ to $\Re$. For the induction step we assume that the defining axiom for $u_{n}$ has been derived in EMA; in particular, this yields that $u_{n}: \Re \rightarrow \Re$ and, hence, by the Mahlo axioms we have for each name $x$ that $(\mathrm{i}) \mathrm{u}_{n+1}(x)$ is a universe containing $x$ and (ii) $\mathrm{u}_{n+1}(x)$ is closed under $\mathrm{u}_{n}$, thus showing that indeed $(n+1)-\mathscr{U}\left(\mathbf{u}_{n+1}(x)\right)$. This concludes our inductive argument.

Further, the linearity and connectivity axioms $\left(\mathscr{U}_{\mathrm{m}}\right.$-Lin) and $\left(\mathscr{U}_{\mathrm{m}}\right.$-Con) of EMA entail the corresponding axioms of $S_{n}$. We have established that $S_{n}$ is a subsystem of EMA for each natural number $n$. 
§3. The wellordering proof for EMA. In this section we will show that $S_{n}$ proves transfinite induction with respect to all types along each initial segment of the ordinal $\varphi(n+1) 00$. This will immediately yield the desired lower bound $\varphi \omega 00$ for EMA. We assume that the reader is familiar with wellordering proofs below the Feferman-Schütte ordinal $\Gamma_{0}$ as they are presented, for example, in Feferman $[4,5]$ or Schütte [25]. Moreover, we presuppose the recent wellordering proofs in the context of metapredicativity in the two papers Jäger, Kahle, Setzer and Strahm [8] as well as Strahm [26].

3.1. Ordinal-theoretic preliminaries. The ordinals which are relevant in the wellordering proofs below are most easily expressed by making use of a ternary Veblen or $\varphi$ function which we are going to define now. The usual Veblen hierarchy generated by the binary function $\varphi$, starting off with the function $\varphi 0 \beta=\omega^{\beta}$ is well known from the literature, c.f., Pohlers [15] or Schütte [25]. The ternary $\varphi$ function is obtained as a straightforward generalization of the binary case by defining $\varphi \alpha \beta \gamma$ inductively as follows:

(i) $\varphi 0 \beta \gamma$ is just $\varphi \beta \gamma$;

(ii) if $\alpha>0$, then $\varphi \alpha 0 \gamma$ denotes the $\gamma$ th ordinal which is strongly critical with respect to all functions $\lambda \xi, \eta . \varphi \delta \xi \eta$ for $\delta<\alpha$.

(iii) if $\alpha>0$ and $\beta>0$, then $\varphi \alpha \beta \gamma$ denotes the $\gamma$ th common fixed point of the functions $\lambda \xi . \varphi \alpha \delta \xi$ for $\delta<\beta$.

For example, $\varphi 10 \alpha$ is $\Gamma_{\alpha}$, and more generally, $\varphi 1 \alpha \beta$ denotes a Veblen hierarchy over $\lambda \alpha . \Gamma_{\alpha}$. It is straightforward how to extend these ideas in order to obtain $\varphi$ functions of all finite arities, and even further to Schütte's Klammersymbole [24].

We let $\Lambda_{3}$ denote the least ordinal greater than 0 which is closed under the ternary $\varphi$ function. In the following we confine ourselves to the standard notation system which is based on this function. Since the exact definition of such a system is a straightforward generalization of the notation system for $\Gamma_{0}$ (c.f., $[15,25]$ ), we do not go into details here. We write $\prec$ for the corresponding primitive recursive wellordering of order type $\Lambda_{3}$ and assume without loss of generality that 0 is the least element with respect to $\prec$. Further, we let Lim denote the primitive recursive set of limit notations and we presuppose a primitive recursively given fundamental sequence $(\ell[n]: n \in \mathbb{N})$ for each limit notation $\ell$; we will assume that $\ell[0]>0$. As the definition of fundamental sequences is easy in the setting of $\varphi$ functions we do not give it here and refer the reader to the relevant proofs in the next paragraph.

There exist primitive recursive functions acting on the codes of our notation system which correspond to the usual operations on ordinals. In the sequel it is often convenient in order to simplify notation to use ordinals and ordinal operations instead of their codes and primitive recursive analogues. Then (for example) $\omega$ and $\omega+\omega$ stand for the natural numbers whose order type with respect to $\prec$ are $\omega$ and $\omega+\omega$.

By making use of the recursion theorem and a little amount of complete induction on the natural numbers one can easily represent all the above primitive recursive notions in EMA and each of its subsystems $S_{n}$. When working in the systems $\mathrm{S}_{n}$ in this section, we let $a, b, c, d, e, \ldots$ range over the field of $\prec$ and $\ell$ denote limit notations. 
Finally, let us put as usual for each $\mathbb{L}$ formula $A(x)$ :

$$
\begin{aligned}
& \operatorname{Prog}(A):=(\forall a)[(\forall b \prec a) A(b) \rightarrow A(a)], \\
& \operatorname{TI}(A, a):=\operatorname{Prog}(A) \rightarrow(\forall b \prec a) A(b) .
\end{aligned}
$$

If we want to stress the relevant induction variable of a formula $A$, we sometimes write $\operatorname{Prog}(\lambda x . A(x))$ and $\operatorname{TI}(\lambda x . A(x), a)$ instead of $\operatorname{Prog}(A)$ and $\operatorname{TI}(A, a)$, respectively. Moreover, we let $\operatorname{Prog}(U)$ and $\operatorname{Prog}(u)$ stand for $\operatorname{Prog}(\lambda x . x \in U)$ and $\operatorname{Prog}(\lambda x . x \dot{\in} u)$, respectively; $\operatorname{TI}(U, a)$ and $\operatorname{TI}(u, a)$ are understood analogously.

3.2. Deriving transfinite inductions in $S_{n}$. In this paragraph we will establish that $S_{n}$ proves $(\forall X) \operatorname{TI}(X, \alpha)$ for each ordinal $\alpha$ less than $\varphi(n+1) 00$. This shows in particular that $\varphi \omega 00$ is a lower bound for the proof-theoretic ordinal of EMA. The key lemma to be proved in the sequel says that if $x$ is a name and we know that transfinite induction holds below $a$ with respect to all types (names) in $\mathbf{u}_{n}\left(\mathrm{u}_{n}(x)\right)$ (i.e., a universe containing a universe that contains $x$ ), then transfinite induction holds even below $\varphi n a 0$ for all types (names) in $\mathrm{u}_{n}(x)$.

MAIN LEMMA 3.1. We have for all natural numbers $n$ that $S_{n}$ proves:

$$
\Re(x) \wedge\left(\forall y \dot{\in} \mathrm{u}_{n}\left(\mathrm{u}_{n}(x)\right)\right) \operatorname{TI}(y, a) \rightarrow\left(\forall y \dot{\in} \mathrm{u}_{n}(x)\right) \operatorname{TI}(y, \varphi n a 0) .
$$

The proof of the main lemma is by (meta) induction on $n$. The case $n=0$ is immediate from the work of Feferman and Schütte on wellordering proofs below $\Gamma_{0}$, c.f., e.g., Feferman [4, 5] and Schütte [25]. The key steps are as follows: given a name $x$ and assuming $\left(\forall y \dot{\epsilon} \mathrm{u}_{0}\left(\mathrm{u}_{0}(x)\right)\right) \mathrm{TI}(y, a)$, we also have $\left(\forall y \dot{\epsilon} \mathrm{u}_{0}\left(\mathrm{u}_{0}(x)\right)\right) \mathrm{Tl}\left(y, \omega^{a+1}\right)$, due to the fact that universes are closed under elementary (and hence arithmetical) comprehension. Further, given an arbitrary name $y$ in $u_{0}(x)$ we can now set up the ordinary (arithmetical) jump hierarchy starting with $y$ below $\omega^{a+1}$ in $u_{0}(x)$; this hierarchy can be described by making use of the recursion theorem and using join at limit stages. The fact that the hierarchy is total or well-defined in $u_{0}(x)$ is shown by induction up to $\omega^{a+1}$ and indeed this is possible since the relevant statement to be established defines a type in $u_{0}\left(u_{0}(x)\right)$, a universe above $u_{0}(x)$, c.f., Lemma 3.2 below for a similar argument. But the existence of the jump hierarchy starting from $y$ below $\omega^{a+1}$ immediately entails $\mathrm{TI}(y, \varphi a 0)$, for example by Lemma 5.3.1 in Feferman [5] or Lemma 10 on p. 187 in Schütte [25]. This ends our brief sketch of the (well-known) assertion of our main lemma in the case $n=0$.

Let us turn to the induction step. For that purpose we fix a natural number $n$ and assume that (1) is true for $n$, aiming at a proof of the assertion of our main lemma for $n+1$. I.e., we want to show in $S_{n+1}$ that for all names $x$,

$$
\left(\forall y \dot{\epsilon} \mathrm{u}_{n+1}\left(\mathrm{u}_{n+1}(x)\right)\right) \mathrm{TI}(y, a) \rightarrow\left(\forall y \dot{\epsilon} \mathrm{u}_{n+1}(x)\right) \mathrm{TI}(y, \varphi(n+1) a 0) .
$$

A crucial ingredient in the proof of (2) are (uniform) transfinite hierarchies of $n$-hyperuniverses within an $(n+1)$-hyperuniverse. Such hierarchies are introduced via the recursion or fixed point theorem. In particular, we let $h_{n}$ be a closed term of $\mathbb{L}$ so that we have provably in EETJ:

$$
\begin{aligned}
\mathrm{h}_{n} x 0 & \simeq \mathrm{u}_{n}(x), \\
h_{n} x(a+1) & \simeq \mathrm{u}_{n}\left(\mathrm{~h}_{n} x a\right), \\
\mathrm{h}_{n} x \ell & \simeq \mathrm{u}_{n}\left(\mathrm{j}\left(\{a: a \prec \ell\}, \mathrm{h}_{n} x\right)\right) .
\end{aligned}
$$


Hence, the hierarchy starts with a $n$-hyperuniverse containing (the name) $x$, at successor stages one puts an $n$-hyperuniverse on top of the hierarchy defined so far, and at limit stages a universe above the disjoint union of the previously defined hierarchy is taken. Of course, in general, one needs some amount of transfinite induction in order to show that $h_{n}$ is well-defined in an $(n+1)$-hyperuniverse $y$. Therefore, in order to express the well-definedness of $h_{n}$ below $a$ in $y$, we let $\operatorname{Hier}_{n}(y, a)$ denote the conjunction of the following three formulas:

(i) $(\forall x \dot{\epsilon} y)(\forall b \prec a)\left(\mathrm{h}_{n} x b \dot{\in} y\right)$,

(ii) $(\forall x \dot{\in} y)(\forall b \prec a) n-\mathscr{U}\left(\mathrm{h}_{n} x b\right)$,

(iii) $(\forall x \dot{\in} y)(\forall b \prec a)(\forall c \prec b)\left(\mathrm{h}_{n} x c \dot{\in} \mathrm{h}_{n} x b\right)$.

The following lemma expresses that $h_{n}$ is well-defined below $a$ in an $(n+1)$ hyperuniverse $u_{n+1}(x)$ provided that transfinite induction below $a$ is available with respect to all types (names) in $\mathrm{u}_{n+1}\left(\mathrm{u}_{n+1}(x)\right)$.

LeMma 3.2. We have that $\mathrm{S}_{n+1}$ proves:

$$
\Re(x) \wedge\left(\forall y \dot{\in} \mathrm{u}_{n+1}\left(\mathrm{u}_{n+1}(x)\right) \operatorname{TI}(y, a) \rightarrow \operatorname{Hier}_{n}\left(\mathrm{u}_{n+1}(x), a\right) .\right.
$$

Proof. Reasoning in $\mathrm{S}_{n+1}$ we assume that $x$ is a name and for all types (names) $y$ in $\mathrm{u}_{n+1}\left(\mathrm{u}_{n+1}(x)\right)$ transfinite induction is available below $a$. We have to show $\operatorname{Hier}_{n}\left(\mathbf{u}_{n+1}(x), a\right)$, i.e., for all $z \dot{\in} \mathbf{u}_{n+1}(x)$,

$$
\begin{aligned}
& (\forall b \prec a)\left(\mathrm{h}_{n} z b \dot{\in} \mathrm{u}_{n+1}(x)\right), \\
& (\forall b \prec a) n-\mathscr{U}\left(\mathrm{h}_{n} z b\right), \\
& (\forall b \prec a)(\forall c \prec b)\left(\mathrm{h}_{n} z c \dot{\epsilon} \mathrm{h}_{n} z b\right) .
\end{aligned}
$$

Since $\left\{b \prec a: h_{n} z b \dot{\in} \mathrm{u}_{n+1}(x)\right\}$ defines a type in $\mathrm{u}_{n+1}\left(\mathrm{u}_{n+1}(x)\right)$ by elementary comprehension, (3) follows by a straightforward transfinite induction. Moreover, (4) is immediate from (3) by the definition of $h_{n}$, the fact that universes consist of names only, and the defining axioms for the $\mathrm{u}_{n}$ 's.

As to (5), we first observe that $\left\{b \prec a:(\forall c \prec b)\left(h_{n} z c \dot{\epsilon} h_{n} z b\right)\right\}$ defines a type in $u_{n+1}(x)$ (and hence in $u_{n+1}\left(u_{n+1}(x)\right)$ by transitivity): to see this one basically applies join to (3) and subsequently uses an obvious instance of elementary comprehension. Given our general assumption, we can now derive (5) by an inductive argument. To show that the above type is progressive with respect to $\prec$ one proceeds straightforwardly in case $b$ is not a limit ordinal. If $b$ is limit and $c \prec b$, then also $c+1 \prec b$ and $h_{n} z c \dot{\in} h_{n} z(c+1)$. On the other hand, one easily sees that there is a name of the universe denoted by $h_{n} z(c+1)$ which belongs to $h_{n} z b$, since we have by definition $\mathrm{j}\left(\{c: c \prec b\}, \mathrm{h}_{n} z\right) \dot{\in} \mathrm{h}_{n} z b$. But then $\mathrm{h}_{n} z c \dot{\in} \mathrm{h}_{n} z b$ is immediate by strong transitivity (Lemma 2.3), which also holds for normal universes in $\mathrm{S}_{n+1}$.

Crucial for the wellordering proof below is the notion $n l_{x}^{c}(a)$ of transfinite induction up to a for all types (respectively names) belonging to a $n$-hyperuniverse $\mathrm{h}_{n} x b$ for $b \prec c$, which is given as follows:

$$
n I_{x}^{c}(a):=(\forall b \prec c)\left(\forall y \dot{\in} \mathbf{h}_{n} x b\right) \operatorname{TI}(y, a) .
$$

The next lemma tells us that $\left.n\right|_{x} ^{\ell}(a)$ can be represented by a type in $h_{n} x \ell$. 
Lemma 3.3. We have that $\mathrm{S}_{n+1}$ proves:

$$
\begin{aligned}
& \Re(x) \wedge \operatorname{Hier}_{n}\left(\mathrm{u}_{n+1}(x), a\right) \rightarrow \\
& \quad\left(\forall y \dot{\epsilon} \mathrm{u}_{n+1}(x)\right)(\forall \ell \prec a)\left(\exists z \dot{\epsilon} \mathrm{h}_{n} y \ell\right)(\forall b)\left[\left.b \dot{\epsilon} z \leftrightarrow n\right|_{y} ^{\ell}(b)\right] .
\end{aligned}
$$

Proof. Working in $\mathrm{S}_{n+1}$, let $x$ be a name and assume $\operatorname{Hier}_{n}\left(\mathrm{u}_{n+1}(x), a\right)$. In addition, fix a name $y$ in $u_{n+1}(x)$ and a limit notation $\ell \prec a$. By the definition of $\mathrm{h}_{n} y \ell$ we have that $\mathrm{j}\left(\{c: c \prec \ell\}, \mathrm{h}_{n} y\right) \dot{\in} \mathrm{h}_{n} y \ell$. By closure of $\mathrm{h}_{n} y \ell$ under join this readily entails that also (a name of) the type

$$
\left\{(c, u, v): c \prec \ell \wedge u \dot{\epsilon} \mathrm{h}_{n} y c \wedge v \dot{\epsilon} u\right\}
$$

belongs to $h_{n} y \ell$. Therefore, by closure of $h_{n} y \ell$ under elementary comprehension, there exists a type (name) $z$ in $h_{n} y \ell$ which satisfies the condition claimed by the lemma.

The following lemma makes crucial use of our general induction hypothesis, i.e., the claim (1) of our Main Lemma 3.1 for $n$.

LEMMA 3.4. We have that $\mathrm{S}_{n+1}$ proves:

$$
\Re(x) \wedge \operatorname{Hier}_{n}\left(\mathrm{u}_{n+1}(x), a\right) \rightarrow\left(\forall y \dot{\epsilon} \mathrm{u}_{n+1}(x)\right)(\forall \ell \prec a) \operatorname{Prog}\left(\lambda b .\left.n\right|_{y} ^{\ell}(\varphi(n+1) 0 b)\right) .
$$

Proof. Assuming that $x$ is a name and $\operatorname{Hier}_{n}\left(u_{n+1}(x), a\right)$, we aim at showing that $\left(\lambda b .\left.n\right|_{y} ^{\ell}(\varphi(n+1) 0 b)\right)$ is progressive for arbitrary $y \dot{\epsilon} \mathrm{u}_{n+1}(x)$ and limit notations $\ell \prec a$. This claim is immediate by an easy inductive argument from

$$
(\forall c)\left[\left.\left.n\right|_{y} ^{\ell}(c) \rightarrow n\right|_{y} ^{\ell}(\varphi n c 0)\right] \text {. }
$$

Towards a proof of (6) assume $\left.n\right|_{y} ^{\ell}(c)$ and fix a $d \prec \ell$. We have to show $\left(\forall z \dot{\in} \mathrm{h}_{n} y d\right) \operatorname{TI}(z, \varphi n c 0)$. Since $\ell$ is limit we also have $d+1 \prec \ell$ and, hence, our assumption yields

$$
\left(\forall z \dot{\epsilon} \mathrm{h}_{n} y(d+1)\right) \mathrm{TI}(z, c) \text {. }
$$

Further, since $\mathrm{h}_{n} y(d+1)=\mathrm{u}_{n}\left(\mathrm{~h}_{n} y d\right)$ and $\mathrm{h}_{n} y d=\mathrm{u}_{n}(w)$ for a suitable name $w$ in the universe $u_{n+1}(x)$, we are now in a position to apply our general assumption (1) for $n$ and obtain

$$
\left(\forall z \dot{\in} h_{n} y d\right) \operatorname{TI}(z, \varphi n c 0) .
$$

Since $d$ was an arbitrary notation less than $\ell$ we thus have shown $\left.n\right|_{y} ^{\ell}(\varphi n c 0)$. This ends our proof of (6).

Now in order to establish $\operatorname{Prog}\left(\lambda b .\left.n\right|_{y} ^{\ell}(\varphi(n+1) 0 b)\right.$, it is clearly enough to show the three claims,

$$
\begin{aligned}
& \left.n\right|_{y} ^{\ell}(\varphi(n+1) 00) \\
& \left.\left.n\right|_{y} ^{\ell}(\varphi(n+1) 0 b) \rightarrow n\right|_{y} ^{\ell}(\varphi(n+1) 0(b+1)) \\
& \left.\left.\operatorname{Lim}(b) \wedge\left(\forall b^{\prime} \prec b\right) n\right|_{y} ^{\ell}\left(\varphi(n+1) 0 b^{\prime}\right) \rightarrow n\right|_{y} ^{\ell}(\varphi(n+1) 0 b) .
\end{aligned}
$$

For (7), observe that we are given a fundamental sequence $z_{v}=\varphi(n+1) 00[v]$ for $\varphi(n+1) 00$, where $z_{0}=1$ and $z_{v+1}=\varphi n z_{v} 0$. Hence, (7) follows from (6) by ordinary (type) induction. The argument for (8) is completely analogous by using the fundamental sequence $z_{v}=\varphi(n+1) 0(b+1)[v]$ for $\varphi(n+1) 0(b+1)$ with $z_{0}=\varphi(n+1) 0 b+1$ and $z_{v+1}=\varphi n z_{v} 0$. Finally, for (9) just observe that if $\operatorname{Lim}(b)$, 
then $\varphi(n+1) 0 b$ is the supremum over $b^{\prime} \prec b$ of $\varphi(n+1) 0 b^{\prime}$, so that the claim is immediate in this case. All together this completes the proof of our lemma.

An important tool in the proof of Lemma 3.6 below is the formula $n \operatorname{Main}_{a}^{x}(b)$. It is the natural adaptation to our setting of similar formulas employed in a wellordering proof below $\Gamma_{0}$ in Feferman [5] and the metapredicative wellordering proofs in Jäger, Kahle, Setzer and Strahm [8] as well as Strahm [26]. Its definition makes use of the binary relation $\uparrow$ on the field of $\prec$,

$$
a \uparrow b:=(\exists c, \ell)(b=c+a \cdot \ell) .
$$

Here of course + and $\cdot$ are the primitive recursive operations corresponding to ordinal addition and multiplication on the field of $\prec$. The formula $n \operatorname{Main}_{a}^{x}(b)$ now has the following definition,

$$
n \operatorname{Main}_{a}^{x}(b):=(\forall y \dot{\in} x)(\forall c, d)\left[d \preceq a \wedge \omega^{1+b} \uparrow d \wedge n l_{y}^{d}(c) \rightarrow n l_{y}^{d}(\varphi(n+1) b c)\right]
$$

Given a name $x$ and assuming $\operatorname{Hier}_{n}\left(\mathrm{u}_{n+1}(x), a\right)$, the following lemma says that the formula $n \operatorname{Main}_{a}^{\mathrm{u}_{n+1}(x)}(b)$ defines a type in the universe $\mathrm{u}_{n+1}\left(\mathrm{u}_{n+1}(x)\right)$. The proof of the lemma is straightforward and very similar in spirit to the proof of Lemma 3.3.

LEMMA 3.5. We have that $\mathrm{S}_{n+1}$ proves:

$$
\begin{aligned}
\Re(x) \wedge & \operatorname{Hier}_{n}\left(\mathrm{u}_{n+1}(x), a\right) \rightarrow \\
& \left(\exists y \dot{\epsilon} \mathrm{u}_{n+1}\left(\mathrm{u}_{n+1}(x)\right)\right)(\forall b)\left[b \dot{\epsilon} y \leftrightarrow n \operatorname{Main}_{a}^{\mathrm{u}_{n+1}(x)}(b)\right] .
\end{aligned}
$$

Proof. Reason in $\mathrm{S}_{n+1}$ and assume that $x$ is a name so that $\operatorname{Hier}_{n}\left(\mathrm{u}_{n+1}(x), a\right)$ holds. In particular, we have for each $z \dot{\epsilon} \mathrm{u}_{n+1}(x)$,

$$
(\forall c \prec a) \mathrm{h}_{n} z c \dot{\in} \mathrm{u}_{n+1}(x) .
$$

Applying join twice to (10) allows us to conclude that (a name of) the type

$$
\left\{(c, u, v): c \prec a \wedge u \dot{\in} \mathrm{h}_{n} z c \wedge v \dot{\in} u\right\}
$$

belongs to the universe $u_{n+1}(x)$ (and hence also to $u_{n+1}\left(u_{n+1}(x)\right)$ ). Since the name of the type (11) is uniformly given in each $z \dot{\in} \mathrm{u}_{n+1}(x)$ we can apply join in the universe $u_{n+1}\left(u_{n+1}(x)\right)$ in order to obtain a name of the type

$$
\left\{(z, c, u, v): z \dot{\epsilon} \mathrm{u}_{n+1}(x) \wedge c \prec a \wedge u \dot{\in} \mathrm{h}_{n} z c \wedge v \dot{\in} u\right\}
$$

in the universe $u_{n+1}\left(u_{n+1}(x)\right)$. But now, clearly, $\left\{b: n \operatorname{Main}_{a}^{u_{n+1}(x)}(b)\right\}$ is given elementarily in the type (12) and, hence, the claim of our lemma is established.

We are now ready to turn to the crucial lemma concerning $n \operatorname{Main}_{a}^{x}(b)$. It is a natural generalization of Main Lemma $I$ in [8] and [26]. Much of the proof is analogous to the proof in [8] and, therefore, we only want to concentrate on the main new points below.

Lemma 3.6. We have that $\mathrm{S}_{n+1}$ proves:

$$
\Re(x) \wedge \operatorname{Hier}_{n}\left(\mathrm{u}_{n+1}(x), a\right) \rightarrow \operatorname{Prog}\left(\lambda b . n \operatorname{Main}_{a}^{\mathrm{u}_{n+1}(x)}(b)\right) .
$$


Proof. Let us work informally in $\mathrm{S}_{n+1}$ and let us assume that $x$ is a name and $\operatorname{Hier}_{n}\left(\mathrm{u}_{n+1}(x), a\right)$. In order to show $\operatorname{Prog}\left(\lambda b . n \operatorname{Main}_{a}^{u_{n+1}(x)}(b)\right)$ it is enough to verify the following three claims $(13)-(15)$ :

$$
\begin{aligned}
& n \operatorname{Main}_{a}^{\mathrm{u}_{n+1}(x)}(0) \\
& n \operatorname{Main}_{a}^{\mathrm{u}_{n+1}(x)}(b) \rightarrow n \operatorname{Main}_{a}^{\mathrm{u}_{n+1}(x)}(b+1), \\
& \operatorname{Lim}(b) \wedge(\forall v \in \mathrm{N}) n \operatorname{Main}_{a}^{\mathrm{u}_{n+1}(x)}(b[v]) \rightarrow n \operatorname{Main}_{a}^{\mathrm{u}_{n+1}(x)}(b) .
\end{aligned}
$$

In the following we only elaborate on (13), since (14) and (15) are proved in literally the same manner as (b) and (c) in the proof of Main Lemma I in [8], except for using the function $\lambda b, c . \varphi(n+1) b c$ instead of $\lambda b, c . \varphi 1 b c$ in [8].

Towards the proof of $n \operatorname{Main}_{a}^{\mathrm{u}_{n+1}(x)}(0)$ we assume that a name $y$ in the universe $u_{n+1}(x)$ and a notation $d \preceq a$ with $\omega \uparrow d$ are given. We have to show

$$
(\forall c)\left[\left.\left.n\right|_{y} ^{d}(c) \rightarrow n\right|_{y} ^{d}(\varphi(n+1) 0 c)\right] .
$$

So let us assume $\left.n\right|_{y} ^{d}(c)$. Since $\omega \uparrow d$ we know that $d$ has the form $d_{0}+\omega \cdot \ell$ for a limit notation $\ell$. Hence, in order to derive $n l_{y}^{d}(\varphi(n+1) 0 c)$ it is sufficient to establish for each natural number $v$,

$$
\left.n\right|_{y} ^{d_{0}+\omega \cdot \ell[v]}(\varphi(n+1) 0 c)
$$

Since $\ell[v]>0$ we have that $d_{0}+\omega \cdot \ell[v]$ is always limit and, hence, by means of Lemma 3.4 , we are in a position to conclude

$$
\operatorname{Prog}\left(\lambda b .\left.n\right|_{y} ^{d_{0}+\omega \cdot \ell[v]}(\varphi(n+1) 0 b)\right)
$$

for each natural number $v$. Furthermore, we obtain from Lemma 3.3 that

$$
\left\{b: n \mathbf{l}_{y}^{d_{0}+\omega \cdot \ell[v]}(\varphi(n+1) 0 b)\right\}
$$

forms a type in the universe $h_{n} y\left(d_{0}+\omega \cdot \ell[v]\right)$ for each natural number $v$. But this means in particular that we can now immediately derive assertion (17) from our assumption $n l_{y}^{d}(c)$ and (18). Hence, we have shown (16) and, therefore, also (13).

This concludes our preparatory work towards a proof of (2) in $S_{n+1}$, which is now immediate. Let $x$ be a name and suppose

$$
\left(\forall y \dot{\in} \mathrm{u}_{n+1}\left(\mathrm{u}_{n+1}(x)\right)\right) \mathrm{Tl}(y, a) .
$$

Given this assumption, it is our aim to derive

$$
\left(\forall y \dot{\epsilon} \mathrm{u}_{n+1}(x)\right) \operatorname{TI}(y, \varphi(n+1) a 0) .
$$

We can assume without loss of generality that $a$ is an $\varepsilon$ number, since universes are closed under arithmetical comprehension. Thus, it is enough to establish

$$
\left(\forall y \dot{\in} \mathrm{u}_{n+1}(x)\right) \operatorname{TI}(y, \varphi(n+1) b 0)
$$

for each $b \prec a$. We fix such a $b$ and observe that we also have $\omega^{1+b} \cdot \omega \prec a$. Further, by our assumption (19), Lemma 3.2 and Lemma 3.6 we have

$$
\operatorname{Prog}\left(\lambda e . n \operatorname{Main}_{a}^{\mathrm{u}_{n+1}(x)}(e)\right) \text {. }
$$


But (22) together with (19), Lemma 3.2 and Lemma 3.5 immediately show that we have $n \operatorname{Main}_{a}^{u_{n+1}(x)}(b)$, i.e., spelled out

$$
\left(\forall y \dot{\in} u_{n+1}(x)\right)(\forall c, d)\left[\left.\left.d \preceq a \wedge w^{1+b} \uparrow d \wedge n\right|_{y} ^{d}(c) \rightarrow n\right|_{y} ^{d}(\varphi(n+1) b c)\right] .
$$

By choosing $c=0$ and $d=\omega^{1+b} \cdot \omega$ in (23) we get

$$
\left.\left(\forall y \dot{\epsilon} \mathrm{u}_{n+1}(x)\right) n\right|_{y} ^{\omega^{1+h} \cdot \omega}(\varphi(n+1) b 0) .
$$

But now one immediately realizes that (24) entails (21). Since $b \prec a$ was arbitrary, we have thus shown (20). This is as desired and ends our proof of (2), given that the assumption (1) of our main lemma holds for $n$. All together this concludes our proof of Main Lemma 3.1.

A straightforward iterated application of Main Lemma 3.1 yields the following crucial theorem about the proof-theoretic lower bound of the theories $S_{n}$.

THEOREM 3.7. We have for all natural numbers $n$ and all ordinals $\alpha$ less than $\varphi(n+1) 00$ that $\mathrm{S}_{n}$ proves $(\forall X) \mathrm{TI}(X, \alpha)$.

Proof. We fix a natural number $n$ and inductively define the fundamental sequence $\left(\alpha_{j}: j \in \mathbb{N}\right)$ for $\varphi(n+1) 00$ by $\alpha_{0}:=1$ and $\alpha_{j+1}:=\varphi n \alpha_{j} 0$. We further use the notation $u_{n}^{(j)}(x)$ for the $j$-fold application of $u_{n}$ to $x$, i.e., $u_{n}^{(0)}(x):=x$ and $\mathrm{u}_{n}^{(j+1)}(x):=\mathrm{u}_{n}\left(\mathrm{u}_{n}^{(j)}(x)\right)$. We have to show that $\mathrm{S}_{n}$ proves $(\forall X) \operatorname{TI}\left(X, \alpha_{k}\right)$ for each natural number $k$. Towards that aim one makes straightforward use of Main Lemma 3.1 in order to show by induction on $j \leq k$ that $S_{n}$ proves

$$
\Re(x) \rightarrow\left(\forall y \dot{\in} \mathrm{u}_{n}^{(k+1-j)}(x)\right) \operatorname{TI}\left(y, \alpha_{j}\right) .
$$

If we choose $j=k$ in assertion (25), then we obtain that $\mathrm{S}_{n}$ derives

$$
\Re(x) \rightarrow\left(\forall y \dot{\in} \mathrm{u}_{n}(x)\right) \mathrm{TI}\left(y, \alpha_{k}\right) .
$$

In particular, (26) entails that $(\forall x)\left(\Re(x) \rightarrow \mathrm{TI}\left(x, \alpha_{k}\right)\right.$ is provable in $\mathrm{S}_{n}$. Since we have an axiom saying that each type has a name, we have thus shown in $S_{n}$ the assertion $(\forall X) \operatorname{TI}\left(X, \alpha_{k}\right)$. This is as desired and ends our proof.

Using the definition of proof-theoretic ordinal from [11] we thus obtain:

COROLLARY 3.8. We have for all natural numbers $n$ that $\varphi(n+1) 00 \leq\left|S_{n}\right|$.

Due to Lemma 2.4 we have now established the desired lower bound for EMA.

Corollary 3.9. $\varphi \omega 00 \leq \mid$ EMA $\mid$.

§4. The wellordering proof for $\mathrm{KPm}^{0}$. In the following let us quickly indicate how the wellordering proofs given in the previous section can be adapted to the context of admissible set theory, namely the theory $\mathrm{KPm}^{0}$. As the procedure is very analogous to EMA we only sketch the main new points and do not spell out the wellordering proof for $\mathrm{KPm}^{0}$ in all details. 
4.1. The theory $\mathrm{KPm}^{0}$ and its subsystems. In this paragraph we briefly review the theory $\mathrm{KPm}^{0}$, and we identify its crucial subsystems $\mathrm{T}_{n}$ corresponding to the subsystems $S_{n}$ of EMA. We refer to Jäger and Strahm [11] for a more detailed exposition of $\mathrm{KPm}^{0}$.

Our version of $\mathrm{KPm}^{0}$ is formulated with the natural numbers as urelements. Accordingly, we let $\mathscr{L}_{1}$ denote the usual language of arithmetic with function and relation symbols for all primitive recursive functions and relations. We further assume that $\mathscr{L}_{1}$ also includes the free anonymous relation symbol Q. The theory $\mathrm{KPm}{ }^{0}$ is now formulated in the extension $\mathscr{L}^{*}=\mathscr{L}_{1}(\in, \mathrm{N}, \mathrm{S}, \mathrm{Ad})$ of $\mathscr{L}_{1}$ by the membership relation symbol $\epsilon$, the set constant $N$ for the set of natural numbers and the unary relation symbols $\mathrm{S}$ and Ad for sets and admissibles, respectively. Terms and formulas of $\mathscr{L}^{*}$ are defined in the standard way; in particular, $\Delta_{0}, \Sigma, \Pi$, $\Sigma_{n}$ and $\Pi_{n}$ denote the obvious classes of $\mathscr{L}^{*}$ formulas. Further, equality between objects is regarded as a defined notion in the expected manner, c.f., [11].

The $\mathscr{L}^{*}$ theory $\mathrm{KPm}^{0}$ is based on classical first order logic with equality. Its non-logical axioms comprise:

(i) the expected ontological axioms regarding the set constant $\mathrm{N}$ and the unary predicates $S$ and Ad for sets and admissibles, respectively; in particular, it is claimed that admissibles are linearly ordered, i.e.,

$$
\operatorname{Ad}(x) \wedge \operatorname{Ad}(y) \rightarrow x \in y \vee x=y \vee y \in x .
$$

(ii) the axioms of Peano arithmetic PA.

(iii) the Kripke Platek axioms, namely pairing, union, separation for $\Delta_{0}$ formulas and collection for $\Delta_{0}$ formulas.

(iv) the Mahlo axioms; these include for all $\Delta_{0}$ formulas $A(x, y, \vec{u})$ whose parameters belong to the list $x, y, \vec{u}$ :

$$
(\forall x)(\exists y) A(x, y, \vec{u}) \rightarrow(\exists z)[\operatorname{Ad}(z) \wedge \vec{u} \in z \wedge(\forall x \in z)(\exists y \in z) A(x, y, \vec{u})]
$$

(v) complete induction on the natural numbers for $\Delta_{0}$ formulas.

Let us now turn to the subsystems $\mathrm{T}_{n}$ of $\mathrm{KPm}^{0}$. In complete analogy to the systems $S_{n}$, the principal set existence axiom of $T_{n}$ claims that each set is contained in an $n$-(hyper)inaccessible set. For each natural number $n$ we use $n$-la $(z)$ in order to express that the set $z$ is $n$-inaccessible; $n$-la $(z)$ is a $\Delta_{0}$ formula of $\mathscr{L}^{*}$ and is inductively given as follows:

$$
\begin{aligned}
0-\operatorname{la}(z) & :=\operatorname{Ad}(z) ; \\
(n+1)-\operatorname{la}(z) & :=\operatorname{Ad}(z) \wedge(\forall x \in z)(\exists y \in z)(x \in y \wedge n-\operatorname{la}(y)) .
\end{aligned}
$$

For each natural number $n$, the $\mathscr{L}^{*}$ theory $\mathrm{T}_{n}$ is now defined to be $\mathrm{KPm}^{0}$ without the Mahlo axioms plus the following limit axiom,

$$
(\forall x)(\exists y)(x \in y \wedge n-\mathrm{la}(y)) .
$$

Hence, $T_{n}$ formalizes an $(n+1)$-inaccessible universe of sets (without foundation). Thus $T_{0}$ is exactly Jäger's well-known set theory $\mathrm{KPi}^{0}$ (c.f., [7]) and $\mathrm{T}_{1}$ is the system $\mathrm{KPh}^{0}$ considered in Strahm [27].

Lemma 4.1 ( $n$-inaccessibles in $\left.\mathrm{KPm}^{0}\right)$. We have that $\mathrm{T}_{n}$ is contained in $\mathrm{KPm}^{0}$ for each natural number $n$. 
PROOF. We verify the assertion of this lemma by induction on $n$. In the case $n=0$ we have to show that each set $u$ is contained in an admissible set. This is immediate by applying $\Pi_{2}$ reflection on admissibles to the $\Pi_{2}$ formula $(\forall x)(\exists y)(u=u)$. For the induction step let us assume that $\mathrm{KPm}^{0}$ includes $\mathrm{T}_{n}$, i.e., in particular, $\mathrm{KPm}^{0}$ proves $(\forall x)(\exists y)(x \in y \wedge n$-la $(y))$. But then one makes use of pairing and the fact that admissibles are transitive to show that we also have, provably in $\mathrm{KPm}^{0}$,

$$
(\forall x)(\exists y)[x \in y \wedge u \in y \wedge n \text {-la }(y)] .
$$

If we apply $\Pi_{2}$ reflection on Ad to this last assertion, then we obtain in $\mathrm{KPm}^{0}$,

$$
(\exists z)[\operatorname{Ad}(z) \wedge u \in z \wedge(\forall x \in z)(\exists y \in z)(x \in y \wedge n-\mathrm{la}(y))] .
$$

This reveals that $\mathrm{KPm}^{0}$ derives $(\forall u)(\exists z)(u \in z \wedge(n+1)-\mathrm{la}(z))$. We have thus shown that $\mathrm{T}_{n+1}$ is contained in $\mathrm{KPm}^{0}$.

4.2. Remarks on deriving transfinite inductions in $T_{n}$. As mentioned above, the wellordering proofs given for the subsystems $S_{n}$ of EMA directly carry over to the subsystems $\mathrm{T}_{n}$ of $\mathrm{KPm}^{0}$. In the sequel we only want to address some delicate points which are characteristic for the set-theoretic framework, without redoing the whole wellordering proof.

We have seen in the previous section that one of the essential tools in the wellordering proof for EMA are transfinite hierarchies of $n$-hyperuniverses. Correspondingly, we have to consider transfinite hierarchies of $n$-inaccessibles in the framework of admissible set theory. For the construction of such hierarchies we need a uniform means for picking an $n$-inaccessible set above any given set. More precisely, we want a $\Sigma_{1}$ operation $(\cdot)^{{ }_{n}}$ of $\mathrm{T}_{n}$ such that provably in $\mathrm{T}_{n}$ we have that for each $u$, the set $u^{t_{n}}$ is an $n$-inaccessible set containing $u$.

Not long ago Gerhard Jäger proved that in $\mathrm{KPi}^{0}$ there exists a $\Sigma_{1}$ operation picking the next admissible set above any set $u$, i.e., the least admissible set containing $u$. It happens that Jäger's proof readily generalizes in order to provide the required operation $(\cdot)^{+_{n}}$ in $\mathrm{T}_{n}$ : one defines $u^{+_{n}}$ to be the least $n$-inaccessible set above $u$, i.e.,

$$
u^{+_{n}}:=\bigcap\{x: u \in x \wedge n-\operatorname{la}(x)\} .
$$

For completeness we give the (adaptation of the) proof of Jägers theorem. It appears that linearity of admissibles is crucial in the argument below.

LemmA 4.2. 1. $T_{n}$ proves that $u^{+_{n}}$ is a set and, in addition, $n$-la $\left(u^{+_{n}}\right)$. Moreover, the operation $u \mapsto u^{+_{n}}$ is $\Sigma_{1}$ definable in $\mathrm{T}_{n}$.

2. We have that 1 . relativizes to any $(n+1)$-inaccessible set.

Proof. In the following we prove the first part of the lemma only; the second part is immediate by relativization. Let us work informally in $\mathrm{T}_{n}$. Given a set $u$, the limit axiom of $T_{n}$ guarantees the existence of a set $y$ such that $n-\operatorname{la}(y)$ and $u \in y$ and, hence, we have that

$$
u^{+n}=\bigcap\{x \in y \cup\{y\}: u \in x \wedge n-\operatorname{la}(x)\}
$$

by linearity of admissibles. This proves that $u^{+_{n}}$ is indeed a set and one readily sees that the operation $u \mapsto u^{+_{n}}$ is $\Sigma_{1}$ definable. 
It remains to show that $u^{+_{n}}$ is $n$-inaccessible, i.e., $n$-la $\left(u^{+_{n}}\right)$. For that purpose we define $u^{++_{n}}:=\left(u^{+n}\right)^{+_{n}}$ and first convince ourselves that

$$
u^{+_{n}} \neq u^{++_{n}}
$$

For a contradiction, assume $u^{+_{n}}=u^{++_{n}}$. By $\Delta_{0}$ separation, we have that $r:=$ $\left\{x \in u^{+_{n}}: x \notin x\right\}$ is a set and, moreover, $r \in z$ for each $n$-inaccessible set $z$ such that $u^{+_{n}} \in z$, i.e., $r \in u^{++_{n}}$ by definition. But then $r \in u^{t_{n}}$ since we have assumed $u^{+_{n}}=u^{++_{n}}$. This yields a contradiction since

$$
r \in r \leftrightarrow r \in u^{+_{n}} \wedge r \notin r \leftrightarrow r \notin r .
$$

Using (27), there exists a set $z$ such that $n$-la(z), $u \in z$ and $u^{+_{n}} \notin z$, and indeed we now show that $z=u^{+_{n}}$. The inclusion $u^{+_{n}} \subset z$ is obvious. In order to establish that $z \subset u^{+_{n}}$ we pick an arbitrary set $x$ with $n-\operatorname{la}(x)$ and $u \in x$ and verify $z \subset x$. By linearity we have $z \in x \vee z=x \vee x \in z$. In case of $z \in x$ or $z=x, z \subset z$ is obvious. But $x \in z$ is impossible since this would imply $u^{+_{n}} \in z$, a contradiction to the choice of $z$. All together we have shown $z=u^{+_{n}}$, which entails $n-\operatorname{la}\left(u^{+_{n}}\right)$ as desired. This finishes our argument. We observe that $\Delta_{0}$ collection was not used in this proof.

Once we have the operations $(.)^{+_{n}}$ at hand we are able to build transfinite hierarchies of $n$-inaccessibles within an $(n+1)$-inaccessible set. It is now a matter of routine to translate the wellordering proofs given in the framework of explicit mathematics for the systems $S_{n}$ to the language of set theory and the systems $T_{n}$. Indeed, some points which needed special attention in explicit mathematics are even simpler in the set-theoretic framework. Hence, we are in a position to state the following lower bound for the proof-theoretic ordinal of the system $T_{n}$.

THEOREM 4.3. We have for all natural numbers $n$ that $\varphi(n+1) 00 \leq\left|T_{n}\right|$.

Lemma 4.1 now immediately entails the desired lower bound for $\mathrm{KPm}^{0}$.

COROLlary 4.4. $\varphi \omega 00 \leq\left|\mathrm{KPm}^{0}\right|$.

§5. Concluding remarks. Let us summarize our results concerning the prooftheoretic ordinals of the theories EMA, $\mathrm{KPm}^{0}, \mathrm{~S}_{n}$, and $\mathrm{T}_{n}$. The lower bounds for these theories have been established according to Corollary 3.8, Corollary 3.9, Theorem 4.3, and Corollary 4.4. The corresponding upper bounds are proved in the paper Jäger and Strahm [11]. Hence, we have the following main result.

THEOREM 5.1. We have the following proof-theoretic ordinals:

1. $|\mathrm{EMA}|=\left|\mathrm{KPm}^{0}\right|=\varphi \omega 00$;

2. $\left|S_{n}\right|=\left|T_{n}\right|=\varphi(n+1) 00$.

If we denote by $\left(\mathbb{L}-I_{N}\right)$ and $\left(\mathscr{L}^{*}-I_{N}\right)$ the schema of complete induction on the natural numbers for all formulas in the language $\mathbb{L}$ and $\mathscr{L}^{*}$, respectively, then the lower bound computations given in this article can be extended in a rather straightforward manner in order to yield $\varphi \varepsilon_{0} 00$ as a proof-theoretic lower bound of the systems EMA $+\left(\mathbb{L}-I_{N}\right)$ and $K \mathrm{Km}^{0}+\left(\mathscr{L}^{*}-I_{N}\right)$. The principal benefit of the induction schema compared to the induction axiom is that one has available $\alpha$-hyperuniverses and $\alpha$-(hyper)inaccessibles for $\alpha$ less than $\varepsilon_{0}$ instead of $n$-hyperuniverses and $n$ (hyper)inaccessibles for $n$ less than $\omega$, respectively. The so-obtained lower bounds 
are sharp according to [11]. Moreover, one establishes the expected ordinals for the subsystems $S_{n}$ and $T_{n}$ augmented by the induction schema.

THEOREM 5.2. We have the following proof-theoretic ordinals:

1. $\left|\mathrm{EMA}+\left(\mathbb{L}-\mathrm{I}_{\mathrm{N}}\right)\right|=\left|\mathrm{KPm}^{0}+\left(\mathscr{L}^{*}-\mathrm{I}_{\mathrm{N}}\right)\right|=\varphi \varepsilon_{0} 00$;

2. $\left|S_{n}+\left(\mathbb{L}-I_{N}\right)\right|=\left|T_{n}+\left(\mathscr{L}^{*}-I_{N}\right)\right|=\varphi(n+1) \varepsilon_{0} 0$.

We finish this article by mentioning very recent work of Christian Rüede, who in his PhD thesis [23] (c.f., also [20, 21]) considers metapredicative subsystems of second order arithmetic which have the same strength as EMA and $\mathrm{KPm}^{0}$. The key principles introduced and analyzed by Rüede are forms of $\omega$ model reflection and transfinite dependent choice.

\section{REFERENCES}

[1] M. J. BEESON, Foundations of Constructive Mathematics: Metamathematical Studies, Springer, Berlin, 1985.

[2] S. Feferman, A language and axioms for explicit mathematics. Algebra and Logic (J. Crossley, editor), Lecture Notes in Mathematics, vol. 450, 1975, pp. 87-139.

[3] - Constructive theories of functions and classes, Logic Colloquium '78 (M. Boffa, D. van Dalen, and K. McAloon, editors), North Holland, 1979, pp. 159-224.

[4] - Iterated inductive fixed-point theories: Application to Hancock's conjecture, The Patras Symposion (G. Metakides, editor), North Holland, 1982, pp. 171-196.

[5] —_, Reflecting on incompleteness, this Journal, vol. 56 (1991), no. 1, pp. 1-49.

[6] S. FEFERMAN and G. JÄGER, Systems of explicit mathematics with non-constructive $\mu$-operator. Part II, Annals of Pure and Applied Logic, vol. 79 (1996), no. 1, pp. 37-52.

[7] G. JÄGER, The strength of admissibility without foundation, this JOURNAL, vol. 49 (1984), no. 3 , pp. $867-879$.

[8] G. JÄGer, R. Kahle, A. Setzer, and T. STRAhm, The proof-theoretic analysis of transfinitely iterated fixed point theories, this JournaL, vol. 64 (1999), no. 1, pp. 53-67.

[9] G. JÄGER, R. KAHLE, and T. STUDER, Universes in explicit mathematics, Annals of Pure and Applied Logic, vol. 109 (2001), no. 3, pp. 141-162.

[10] G. JÄGER and T. STRAHM, Fixed point theories and dependent choice, Archive for Mathematical Logic, vol. 39 (2000), pp. 493-508.

[11] - Upper bounds for metapredicative Mahlo in explicit mathematics and admissible set theory, this JournaL, vol. 66 (2001), no. 2, pp. 935958.

[12] G. JäGER and T. STUDER, Extending the system $\mathrm{T}_{0}$ of explicit mathematics: The limit and Mahlo axioms, Annals of Pure and Applied Logic, to appear.

[13] R. KaHLE, Applikative theorien und frege-strukturen, Ph.D. thesis, Institut für Informatik und angewandte Mathematik, Universität Bern, 1997.

[14] M. Marzetta, Predicative theories of types and names, Ph.D. thesis, Institut für Informatik und angewandte Mathematik, Universität Bern, 1993.

[15] W. Pohlers, Proof Theory: An introduction, Lecture Notes in Mathematics, vol. 1407, Springer, Berlin, 1989.

[16] M. Rathjen, Proof-theoretic analysis of KPM, Archive for Mathematical Logic, vol. 30 (1991), pp. $377-403$.

[17] - Collapsing functions based on recursively large ordinals: A wellordering proof for KPM, Archive for Mathematical Logic, vol. 33 (1994).

[18] - The strength of Martin-Lof type theory with a superuniverse. Part I, Archive for Mathematical Logic, vol. 39 (2000), no. 1, pp. 1-39.

[19] - The strength of Martin-Löf type theory with a superuniverse. Part II, Archive for Mathematical Logic, vol. 40 (2001), no. 3, pp. 207-233.

[20] C. RÜEDE, Metapredicative subsystems of analysis, Submitted for publication.

[21] - The proof-theoretic analysis of $\Sigma_{1}^{1}$ transfinite dependent choice, Submitted for publication.

[22] — Universes in metapredicative analysis, Submitted for publication. 
[23] — Metapredicative subsystems of analysis, Ph.D. thesis, Institut für Informatik und angewandte Mathematik, Univeristät Bern, 2000.

[24] K. SCHÜTTE, Kennzeichnung von ordnungszahlen durch rekursiv erklärte funktionen, Mathematische Annalen, vol. 127 (1954), pp. 15-32.

[25] - Proof Theory, Springer, Berlin, 1977.

[26] T. Strahm, First steps into metapredicativity in explicit mathematics, Sets and Proofs (S. B. Cooper and J. Truss, editors), Cambridge University Press, 1999, pp. 383-402.

[27] - Autonomous fixed point progressions and fixed point transfinite recursion, Logic Colloquium '98 (S. Buss, P. Hájek, and P. Pudlák, editors), Lecture Notes in Logic, vol. 13, Association for Symbolic Logic, 2000, pp. 449-464.

[28] A. Troelstra and D. van Dalen, Constructivism in Mathematics, vol. I, North Holland, Amsterdam, 1988.

\section{UNIVERSITÄT BERN \\ INSTITUT FÜR INFORMATIK UND ANGEWANDTE MATHEMATIK \\ NEUBRÜCKSTRASSE 10, CH-3012 BERN, SWITZERLAND}

E-mail: strahm@iam.unibe.ch 